Huerta-Soto, R., Guzmán-Avalos, M., Flores-Albornoz, J. \& Tomás-Aguilar, S. (2022). Competencias digitales de los profesores universitarios durante la pandemia por covid-19 en el Perú. Revista Electrónica Interuniversitaria de Formación del Profesorado, 25(1), 49-60.

DOI: https://doi.org/10.6018/reifop.500481

\title{
Competencias digitales de los profesores universitarios durante la pandemia por covid-19 en el Perú
}

\author{
Rosario Huerta Soto ${ }^{(1)}$, Magna Guzmán Avalos ${ }^{(1)}$, Judith Flores Albornoz ${ }^{(1)}$, Segundo Tomas \\ Aguilar $^{(2)}$ \\ ${ }^{(1)}$ Universidad Nacional Santiago Antúnez de Mayolo, Perú. ${ }^{(2)}$ Universidad Cesar Vallejo, Perú.
}

\section{Resumen}

Las tecnologías de la información y la comunicación han tenido un impacto significativo en la calidad de vida de las personas en los últimos años. Pero, su potencial educativo aún no se ha realizado plenamente. A raíz del brote de covid-19, en este artículo se presenta un examen de la competencia digital de los profesores universitarios. Se estudió el desarrollo de estos talentos entre los profesores universitarios. Para recopilar datos, se utilizó Google Forms para crear encuestas en línea. Se enviaron solicitudes a los 240 profesores de la Universidad Nacional Santiago Antunez de Mayolo a través del correo electrónico institucional, y las respuestas de los 187 profesores se incluyeron en la base de datos SPSS V 26 como resultado del brote. Los hallazgos muestran que los profesores universitarios tienen suficientes habilidades digitales, pero su uso en aulas no presenciales está restringido, lo que requiere una revisión de los programas de capacitación en instituciones públicas en este contexto de epidemia de COVID-19.

\section{Palabras clave}

Educación superior; habilidades digitales; clases virtuales; covid-19; pandemia; profesor universitario. 


\title{
Digital competences of university professors during the covid- 19 pandemic in Peru
}

\begin{abstract}
Information and communication technologies have had a significant impact on people's quality of life in recent years. But, its educational potential has yet to be fully realized. In the wake of the covid-19 outbreak, this article presents an examination of the digital competence of university professors. The development of these talents among university professors was studied. To collect data, Google Forms was used to create online surveys. Requests were sent to the 240 professors of the Universidad Nacional Santiago Antunez de Mayolo via institutional email, and the responses of the 187 professors were included in the SPSS V26 database as a result of the outbreak. The findings show that university professors have sufficient digital skills, but their use in non-face-to-face classrooms is restricted, requiring a review of training programs in public institutions in this context of the COVID-19 epidemic.
\end{abstract}

\section{Key words}

Covid-19; digital skills; professor; higher education; pandemic; virtual classes.

\section{Introducción}

En las últimas décadas, la sociedad ha experimentado importantes cambios sociales, económicos y culturales, en los que las Tecnologías de la Información y la Comunicación (TIC) han desempeñado un rol sustancial para la mejora tanto de la calidad de vida de las personas como de las sociedades (Cuervo et al., 2018). Sin embargo, su potencial en la educación todavía se está desarrollando (Hernandez, 2017). A este respecto, la Unión Europea, desde 1993, ha hecho hincapié en la educación, como quedó claro en el informe «Europa y la Sociedad Mundial de la Información», que sostiene la necesidad de formación y alfabetización de los docentes en las TIC

Recientemente, la (UNESCO, 2008) ha publicado el Marco de Competencia TIC para docentes, que pone de relieve la necesidad de formación tecnológica para que los profesores se vuelvan más competitivas de en su desarrollo profesional. Formar docentes en TIC implica profesionalizar su figura, incorporando competencias profesionales deseables para optimizar su desempeño profesional (Michos y Hernández-Leo, 2020). Estamos inmersos en la era digital, el profesorado necesita demostrar un alto nivel de conocimientos tecnológicos para poder desarrollar su trabajo. Teniendo en cuenta la dificultad de gestionar la tecnología educativa, combinando conocimientos educativos y tecnológicos, los docentes podrán hacer frente a las exigencias de su rol profesional (Rodríguez et al., 2021).

A su vez, este conocimiento tecnológico coloca a la tecnología como un aliado, donde puede encontrar 'un socio fiel' para superar las debilidades educativas de su formación general (Hernandez, 2017). La literatura proporciona la necesidad de una mejor formación en cuanto a las habilidades y actitudes obligatorios para promover el aprendizaje de los estudiantes en un mundo tecnológico en crecimiento (Fernández et al., 2018). Así, se hace hincapié en la necesidad de incorporar al contexto educativo todas las herramientas y posibilidades de las tecnologías educativas, con el objetivo de diseñar y desarrollar procesos de enseñanza y 
aprendizaje acordes a las necesidades que emanan de los estudiantes en un mundo de cambios constantes (Santana y Perez, 2020).

La formación de docentes en TIC sigue siendo un desafío tanto para los docentes en formación como para los prestan servicios actualmente (Van Laar et al., 2017). Eclipsado por la importancia de la formación por competencias y amparado por un paradigma más constructivista, se requiere un profesorado competente, no solo pedagógicamente, sino también a nivel tecnológico (Fernández et al., 2018). Las habilidades en TIC de los profesores pueden establecerse como uno de los principales puntos de referencia para los programas de formación de profesores (Grinberga-Zalite et al., 2019). Por lo tanto, se han desarrollado varias terminologías de TIC estándar, utilizando diferentes términos, como alfabetización en TIC, alfabetización digital y competencia en TIC (Reis et al., 2019). Las competencias en TIC se refieren al uso técnico de las TIC, mientras que las competencias en TIC se conceptualizan en términos de uso integrado y funcional de conocimientos, habilidades y actitudes digitales (Hernandez, 2017). En este artículo utilizamos el término competencia digitales porque ofrece una visión más amplia del uso de la tecnología.

Hay un punto importante a tener en cuenta en la búsqueda para analizar este tema. Si bien el concepto de alfabetización digital tiende a usarse globalmente, en el contexto latinoamericano el concepto de competencia digital se usa de manera similar (Cuervo et al., 2018). Este aspecto clave justifica por qué algunos autores hablan de alfabetización digital y competencia digital, de forma indiscriminada (Vega et al., 2017). Estos dos términos no tienen el mismo significado ni el mismo nivel de abstracción. La alfabetización digital es el principio hacia la competencia digital (Reis et al., 2019; Yslado et al., 2021). Por ejemplo, la (UNESCO, 2008) propuso su propia definición sobre competencias digitales, en el que se establece tres niveles: la primera considera la alfabetización digital, seguida de profundización de conocimientos, y la última fase de la gestión eficiente del conocimiento.

Por otro lado, las competencias digitales han evolucionado durante la última década desde una etapa de preparación deseable hasta un nivel de obligatoriedad (Hernandez, 2017). Las habilidades digitales en docentes universitarios han evolucionado desde una perspectiva opcional para los ciudadanos de la sociedad del conocimiento hasta que se ha convertido en una formación obligatoria que los docentes deben adquirir (Terreni y Vilanova, 2019). Además, destaca que la mayoría de los estudios realizados sobre estos temas, revelan una falta de formación en TIC del docente y una formación insuficiente en competencias digitales (Quezada et al., 2020). Por estas razones, se ha reconocido la importancia del desarrollo de estas competencias para los docentes universitarios (Ramirez et al., 2020), podríamos decir que, a pesar de la existencia de estas diversas habilidades para los docentes en América Latina, no ha habido un adecuado reconocimiento de las competencias digitales, sino que ha ganado terreno un mimetismo de las habilidades en TIC (Levano et al., 2017). La situación en Perú, sin embargo, provoca una gran brecha en los programas y planes de aprendizaje aplicado, de ahí que se observa una transición desigual de competencias entre universidades y planes de creación de capacidades digitales en el país. Uno de los resultados de esto es que la falta de experiencia en la formación en competencias digitales.

La enseñanza remota de emergencia se desarrolló como una respuesta rápida a la situación y su naturaleza ha dificultado la adquisición adecuada y el acceso a la tecnología necesaria (Portillo et al., 2020). Esta nueva normalidad exige que cada estudiante y docente esté adecuadamente formado para adquirir las competencias digitales que necesita en entornos digitales (Rodríguez et al., 2021). Debemos recordar que la enseñanza remota de emergencia es una forma alternativa de enseñanza debido a las circunstancias de la crisis sanitaria, mientras que el aprendizaje en línea o mixto de calidad requiere un diseño y una planificación instruccionales cuidadosos (Rodríguez-García et al., 2019). El aprendizaje en línea ha 
demostrado su eficacia en numerosos estudios de investigación siempre que se adopta un modelo sistemático de diseño y desarrollo. Por tanto, para contribuir a la mejora de la calidad de la educación, parece necesario realizar un análisis en profundidad de lo que se ha hecho y lo que se debe mejorar. Este inesperado desafío ha colocado a las universidades como los principales líderes educativos, en la necesidad de desarrollar la digitalización de la educación superior (Sánchez-Cruzado et al., 2021) y ojalá reducir la brecha digital, la brecha social y la desigualdad de género en la población como uno de los ejes para garantizar una educación universitaria de calidad.

Las competencias digitales de los docentes han sido estudiadas por investigadores de tecnología educativa durante varios años. Además, se describe lo que significa para los educadores en todas las etapas ser digitalmente competentes. (Pérez y Rodríguez, 2016) detalla 22 competencias organizadas en cinco áreas y distingue cinco niveles a lo largo de los cuales normalmente se desarrolla la competencia digital de los educadores. Para cada una de las 22 competencias, se proporcionan descriptores de nivel y declaraciones de competencia que permiten a los educadores comprender su nivel de competencia y sus necesidades específicas de desarrollo.

Según Sánchez-Cruzado et al. (2021) las dimensiones que componen las competencias digitales para docentes son cinco: (i) Información: para demostrar la búsqueda e identificación, saber organizar y resguardar la información, considerando su relevancia y propósito para la enseñanza. (ii) Comunicación: para comunicarse y colaborar en los entornos digitales, además es una forma de compartir herramientas digitales, también se considera la interactuación en comunidades digitales. (iii) Creación de contenidos: diseñar, editar e integrar nuevos contenidos y reconstruir contenidos previos para elaborar productos multimedia para los estudiantes. (iv) Seguridad: para la protección de los datos personales, el manejo adecuado de la identidad digital, la protección mediante un antivirus de la información de su computadora, además del nivel de conciencia de la responsabilidad sobre la información que uno comparte. (v) Solución de problemas: identificar necesidades en el uso de recursos digitales, informar decisiones sobre la aplicación de herramientas digitales más adecuada en función a su propósito, resolver problema conceptual a través herramientas digitales, uso la tecnología de manera creativa y resolver problemas técnicos.

En este escenario, se desarrolla un análisis descriptivo de la percepción del docente universitario sobre sus competencias digitales en el ámbito de la pandemia por covid-19. Donde todas las universidades están aplicando metodologías de enseñanza que necesitan de las competencias digitales entre los docentes, con el objetivo de garantizar el buen desarrollo de las labores académicas en este periodo que no será corto. Además, el dominio de las competencias digitales permite implantar las clases no presenciales en universidades públicas. El análisis y diagnóstico de la percepción en docente universitario de sus competencias digitales apoyara medir el efecto del diplomado en competencias digitales del siglo XXI realizado en la Universidad Nacional Santiago Antúnez de Mayolo (UNASAM), durante los meses julio a noviembre del 2020 en pleno confinamiento por consecuencia de la pandemia por covid-19, El diplomado dirigido a 250 docentes universitario comprendido en 3 niveles Nivel I: Inducción ( 2 cursos) ; Nivel II: Mejora continua (4 cursos) y Nivel III: Especialización con 2 cursos..

\section{Metodología}

Se utilizó la metodología exploratoria de enfoque cuantitativo para una investigación de tipo descriptivo- correlacional (Supo y Zacarias, 2020), para medir las competencias digitales del docente universitario se adaptó el cuestionario propuesto por Pérez y Rodríguez, (2016) 
utilizado en profesores de educación primaria en España. El instrumento cuanta con 22 ítems dividido en cinco dimensiones como son: información, comunicación, creación de contenidos, seguridad y solución de problemas. se asignaron valores bajo la escala de Likert; Nulo (1), Bajo (2), Suficiente (3), Elevado (4) y Óptimo (5). Por otro lado, se realizó la validez de contenido, a través de la evaluación de tres jueces, seguido de un análisis de fiabilidad mediante el alfa de Cronbach, se obtuvo un valor de 0.918 , el cual muestra un alto grado de consistencia interna.

En cuanto al proceso de recopilación de datos, debido a la situación de confinamiento por la pandemia se enviaron correos electrónicos a 240 participantes mediante la herramienta online (Goolgle forms) pero solo 199 respondieron de las cuales 12 estuvieron incompletos y finalmente quedaron 187 encuestas válidas.

\section{Resultados}

Para identificar los niveles de las competencias digitales se desarrolló el análisis de fiabilidad, análisis descriptivo de los datos.

\section{Tabla 1.}

Información socio demográfica

\begin{tabular}{ccc}
\hline Variables & Descripción & Porcentaje \\
\hline \multirow{3}{*}{ Edad } & menos a 50 & 33,3 \\
& $51-59$ & 36,8 \\
& 60 a mas & 29,9 \\
& Total & 100,0 \\
\hline \multirow{3}{*}{ Género } & Masculino & 77,0 \\
& Femenino & 23,0 \\
& Total & 100,0 \\
\hline \multirow{3}{*}{ Estado civil } & Solteros & 11,5 \\
& Casado & 57,5 \\
& Conviviente & 17,2 \\
& Separado & 13,8 \\
& Total & 100,0 \\
\hline \multirow{2}{*}{ Condición } & Nombrado & 71,3 \\
& Contratado & 28,7 \\
& Total & 100,0 \\
\hline
\end{tabular}

Fuente: elaboración propia.

La mayoría de los docentes están entre 51 y 59 años, son de sexo masculino, casados y nombrados.

Como se aprecia en la tabla 2, a través del Alfa de Cronbach, la cifra 0.928 indica que el instrumento utilizado es fiable (Hernández \& Mendoza, 2018). La cifra 21 indica el número de preguntas pertenecientes a cada variable observable utilizada para el estudio. 
Tabla 2.

Estadísticas de fiabilidad

\begin{tabular}{lcc}
\hline \multicolumn{1}{c}{ Dimensiones / Variable } & Alfa de Cronbach & N de elementos \\
\hline Información & 0,717 & 3 \\
Comunicación & 0,774 & 6 \\
Creación de contenidos & 0,665 & 4 \\
Seguridad & 0,775 & 4 \\
Solución de problemas & 0,763 & 4 \\
Competencias digitales & 0,928 & 21 \\
\hline
\end{tabular}

Fuente: elaboración propia

Tabla 3.

Estadística descriptiva por ítems

\begin{tabular}{|c|c|c|c|}
\hline $\mathrm{N}^{\circ}$ & ítems & Media & Desviación estándar \\
\hline 1 & Inf_1 & 3,37 & 1,304 \\
\hline 2 & Inf_2 & 3,14 & 1,058 \\
\hline 3 & Inf_3 & 3,53 & 1,228 \\
\hline 4 & Comu_1 & 3,25 & 1,164 \\
\hline 5 & Comu_2 & 3,72 & 1,245 \\
\hline 6 & Comu_3 & 3,80 & 1,247 \\
\hline 7 & Comu_4 & 3,78 & 1,125 \\
\hline 8 & Comu_5 & 3,60 & 1,105 \\
\hline 9 & Comu_6 & 3,75 & 1,143 \\
\hline 10 & Creac_1 & 3,49 & 1,190 \\
\hline 11 & Creac_2 & 3,20 & 1,170 \\
\hline 12 & Creac_3 & 3,34 & 1,119 \\
\hline 13 & Creac_4 & 3,39 & 1,165 \\
\hline 14 & Seg_1 & 3,54 & 1,169 \\
\hline 15 & Seg_2 & 3,94 & 1,135 \\
\hline 16 & Seg_3 & 3,79 & 1,132 \\
\hline 17 & Seg_4 & 3,63 & 1,036 \\
\hline 18 & Soluc_1 & 3,25 & 1,081 \\
\hline 19 & Soluc_2 & 3,30 & 1,286 \\
\hline
\end{tabular}




\begin{tabular}{llll}
20 & Soluc_3 & 3,22 & 1,115 \\
22 & Soluc_4 & 3,43 & 1,187 \\
\hline
\end{tabular}

Fuente: elaboración propia

De acuerdo con la anterior, los ítems mejor valorados son: 15 Proteges tus datos personales y tu identidad digital y 6 Participas y comunicas en entornos digitales. Por otro lado, los de menos valorados fueron el 2 Almacenas información digital y 11 Editas y elaboras recursos educativos.

\section{Tabla 4.}

Estadística descriptiva por dimensiones

\begin{tabular}{lccccc}
\hline Dimensiones / Variable & ítems & Media & Desviación estándar & Mínimo & Máximo \\
\hline Información & 3 & 3,34 & 1,0274 & 1,00 & 4,15 \\
Comunicación & 7 & 3,65 & 0,9771 & 1,00 & 4,50 \\
Creación de contenidos & 4 & 3,36 & 1,2491 & 1,00 & 4,20 \\
Seguridad & 4 & 3,73 & 0,8260 & 1,00 & 4,75 \\
Solución de problemas & 4 & $\mathbf{2 , 4 3}$ & 0,9016 & 1,00 & 4,10 \\
Competencias digitales & 21 & 3,51 & 0,7781 & 1,00 & 4,51 \\
\hline
\end{tabular}

Fuente: elaboración propia

De manera similar la dimensión mejor calificada fue: Seguridad (3.73), y contrariamente el de menor puntaje fue: solución de problemas (2.43).

\section{Figura 1}

Dimensiones de competencias digitales

\section{Dimensiones de competencias digitales}

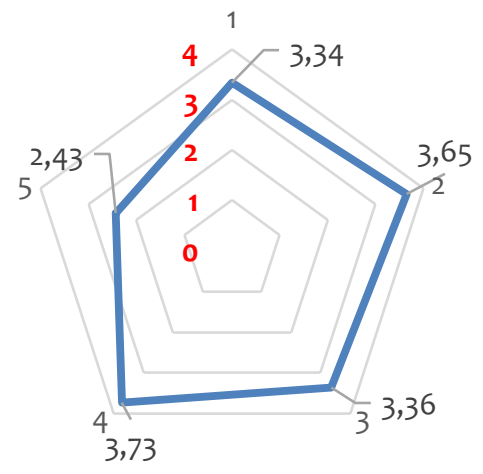


En la figura 1 se puede observar que la dimensión mejor calificada es la seguridad (3.73) y de las cinco dimensiones cuatro están sobre la media de 2.5, esto quiere decir que solo la dimensión solución de problemas necesita ser desarrolladas para mejorar las competencias digitales de los docentes universitarios.

Se han utilizado los puntos de corte del percentil 30 y 70 a partir de la escala para categorizar cada dimensión, según la tabla 5 consta de tres niveles, insuficiente, suficiente y nivel para innovar.

Tabla 5.

Baremos de las dimensiones

\begin{tabular}{|c|c|c|c|}
\hline \multirow{2}{*}{ Variable / dimensión } & \multicolumn{3}{|c|}{ Nivel } \\
\hline & Insuficiente & Suficiente & Para innovar \\
\hline Competencias digitales & 25 a 72 & 73 a 83 & 84 a 91 \\
\hline Información & 3 a 9 & 10 a 12 & 13 a 14 \\
\hline Comunicación & 7 a 22 & 23 a 24 & 25 a 27 \\
\hline Creación de contenidos & 4 a 12 & 13 a 15 & 16 a 17 \\
\hline Seguridad & 4 a 14 & 15 a 17 & 18 a 19 \\
\hline Solución de problemas & 4 a 11 & 12 a 16 & 17 a 18 \\
\hline
\end{tabular}

Fuente: elaboración propia.

Según Pérez y Rodríguez, (2016) las competencias digitales se clasifican en tres niveles de percepción en el ámbito de las competencias digitales: Insuficiente: las competencias evaluadas para permitir la aplicación pedagógica en el aula del profesor universitario son insuficientes. En consecuencia, el docente no podrá enseñar o incorporar estas habilidades en el proceso de enseñanza-aprendizaje (Levano et al., 2017). Suficiente: el docente ha considerado que las competencias digitales son tecnológicamente capaces, pero no suficientes para la innovación en su educación (Reis et al., 2019). Nivel de innovación: los docentes se preocupan por las habilidades técnicas y las competencias digitales, y las nuevas habilidades se utilizan y adaptan a su aprendizaje profesional y los procesos digitales son innovadores para la educación superior (Villarreal-Villa et al., 2019).

Los resultados en la figura 2 indican un nivel insuficiente a la hora de solucionar problemas (40\%), en un nivel suficiente se encuentra la administración de información digital (40\%), comunicación y colaborar en equipo en canales digitales (52\%) y creación de contenidos (37\%) complementando la única dimensión en el nivel para innovar se encuentra la seguridad y elegir software apropiado (34\%). Finalmente, el nivel de las competencias digitales en general se encuentra en suficiente (36\%) en los docentes universitarios. 


\section{Figura 2}

Niveles de competencias digitales.

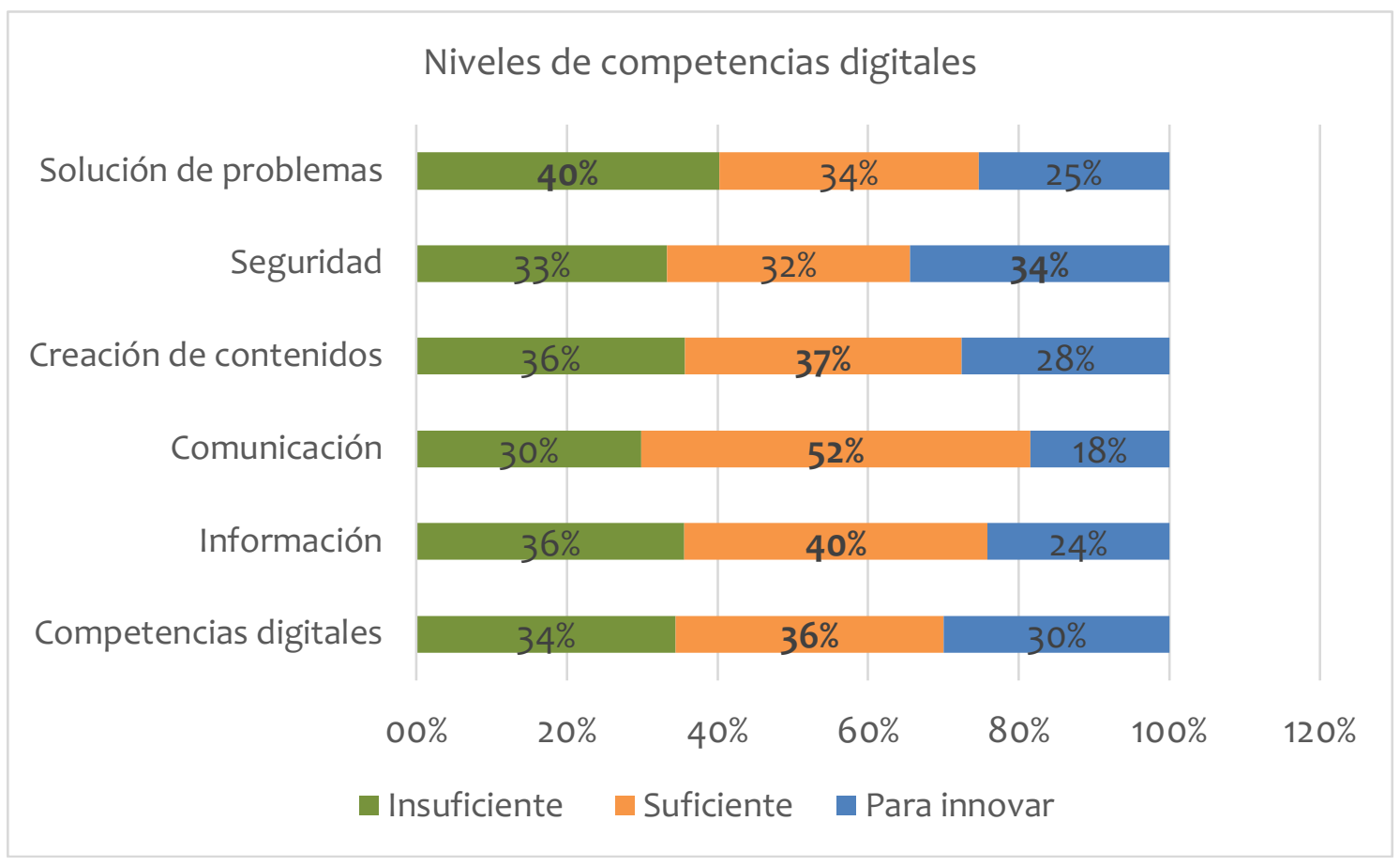

\section{Discusión}

Según Martínez-Garcés y Garcés-Fuenmayor, (2020), en la última década la investigación en el campo educativo de las competencias digitales ha avanzado debido a la introducción de esta. Como ha demostrado Vargas-Murillo, (2019), la comprensión de las competencias digitales de los docentes crece a medida que aumenta la capacidad de las TIC. Los estudios en el escenario de la educación universitaria sobre las competencias digitales están tomando importancia debido al desarrollo y el acceso a las tecnologías (Reis et al., 2019). El estudio de la percepción de las competencias digitales de los docentes universitarios adquiere trascendencia porque el grado de competencia permite aplicar las TIC en las clases no presenciales y en las aulas virtuales (Quezada et al., 2020; Yslado et al., 2021).

El análisis revela que, si el propio docente no tiene desarrollado sus competencias digitales, no solo necesario sino especializado, no puede mejorar las competencias digitales de sus estudiantes (Martínez-Garcés y Garcés-Fuenmayor, 2020). Los docentes tendrían un nivel de suficiente de las competencias digitales, es decir, para instruir un tema de manera efectiva es necesario haber dominado las habilidades de manejo de información digital (Spante et al., 2018). Si bien esta investigación tiene sus limitaciones debido a la muestra y la modalidad de aplicación del instrumento, los hallazgos son una prueba empírica importante sobre la escasez de docentes con suficientes competencias digitales para innovar en el aula, parece ser un requisito no resuelto por las universidades públicas.

Los resultados complementan otros estudios en competencias digitales sobre los docentes universitarios: entre otros, (Villarreal-Villa et al., 2019; Martínez-Garcés y Garcés-Fuenmayor, 2020), amplían el abanico considerable de competencias digitales y refuerzan la necesidad de una intervención urgente para paliar estas deficiencias basadas en la investigación científica. 
Como señalan Pozos y Tejada, (2018), no solos existe un insuficiente manejo de la tecnología dentro de las universidades públicas, sino también la falta de aplicación de las habilidades técnicas para el incremento de competencias en los estudiantes tan importantes para su desenvolvimiento en el futuro (Bond et al., 2019).

El nivel de las competencias digitales de los docentes universitarios está calificado como suficiente, esto debido a que el desarrollando de estas competencias han sido forzados por la implementación de las clases no presenciales desde el semestre 2020-II, esta situación se presenta debido el confinamiento por la pandemia. Asimismo, existen diferencias entre las dimensiones la mejor desarrollada es la seguridad de información y por el contrario la menos desarrolladas es la solución de problemas digitales, por lo cual se hace necesario implementar capacitaciones continuas en este sentido.

Las otras dimensiones están consideradas en el nivel de suficiente, esto permitirá la colaboración para la creación de contenidos digitales y así mejorar el desempeño docente en este escenario de pandemia. Demostrándose que la inclusión de las TIC en las universidades no está exenta de dificultades y desafíos (Hernandez, 2017). Son estas dimensiones involucradas en la efectividad de implementar procesos pedagógicos, apoyados en metodologías y herramientas tecnológicas e interactivas.

En cuanto al cuestionario, queda una interesante línea de investigación centrada en el estudio de la evaluación de la competencia digital percibida. Además, se sugieren nuevas investigaciones en universidades privadas y en docentes de otros países para realizar un análisis comparativo, por otro lado, se sugiere aplicar el mismo cuestionario luego de seis meses para conocer evaluar las competencias digitales en los mismos docentes y obtener un mejor entendimiento de estas variables en este contexto de pandemia.

\section{Conclusiones}

Se ha identificado un nivel suficiente de la percepción del docente universitario sobre sus competencias digitales en el ámbito de la pandemia por covid-19 luego de terminado el diplomado en competencias digitales. Por otro lado, la dimensión más valorada es la relacionada con la seguridad de información, es decir los docentes dominan el resguardo de su información digital generada y entregada a los estudiantes. Acompañado con el conocimiento sobre estas herramientas, el tiempo para explorarlas, el acceso que tienen y el apoyo recibido de parte de la institución.

Contrariamente la dimensión menos valorada es el uso de las competencias digitales para solucionar problemas, esto debido a la limitada disponibilidad de una buena conexión a internet y equipos informáticos modernos por parte de los docentes universitarios, esto es una realidad innegable en las universidades públicas.

\section{Referencias}

Bond, M., Zawacki-Richter, O. \& Nichols, M. (2019). Revisiting five decades of educational technology research: A content and authorship analysis of the British Journal of Educational Technology. British Journal of Educational Technology. 50(1). 12-63. https://doi.org/10.1111/bjet.12730

Cuervo, T., N. Orviz, S. Arce, \& I. Fernández (2018). Tecnoestrés en la Sociedad de la Tecnología y la Comunicación: Revisión bibliográfica a partir de la Web of Science. 
Archivos de Prevención de Riesgos Laborales. 21(1). 18-25. https://dx.doi.org/10.12961/aprl.2018.21.01.4

Fernández, E., Leiva, J. J. \& López, E. (2018). Competencias digitales en docentes de Educación Superior. Revista digital de investigación en docencia universitaria, 12(1). 213231. http://dx.doi.org/10.19083/ridu.12.558

Fernández, F. J., Fernández, M. J. \& Rodríguez, J. M..(2018). El proceso de integración y uso pedagógico de las TIC en los centros educativos madrileños. Educacion XX1, 21(2).: 395-416. https://dx.doi.org/10.5944/educXX1.17907

Grinberga-Zalite, G., Rivza, B., Zvirbule, A. \& Tihankova, T. (2019). Promoting Digital Skills in Higher Education to Strengthen the Competitiveness of the EU Human Capital. International Multidisciplinary Scientific GeoConference: SGEM, 19(5.4). 259-266. http://dx.doi.org/10.5593/sgem2019/5.4/S22.035

Hernandez, R. M. (2017). Impacto de las TIC en la educación: Retos y Perspectivas. Propósitos y representaciones, 5(1). 325-347. http://dx.doi.org/10.20511/pyr2017.v5n1.149

Hernández, R., \& Mendoza, C. (2018). Metodología de la investigación: Las rutas cuantitativa, cualitativa y mixta. Mexico: Mc Graw Hill.

Levano, L., Sánchez, S., Guillén, P., Tello, S., Herrera, N. \& Collantes, Z. (2017). Competencias digitales y educación. propósitos y representaciones, 7(2). 569-588. http://dx.doi.org/10.20511/pyr2019.v7n2.329

Martínez-Garcés, J. \& Garcés-Fuenmayor, J. (2020). Competencias digitales docentes y el reto de la educación virtual derivado de la covid-19. Educación y Humanismo, 22(39). 1-16. https://doi.org/10.17081/eduhum.22.39.4114

Michos, K., \& Hernández-Leo, D. (2020). CIDA: A collective inquiry framework to study and support teachers as designers in technological environments. Computers \& Education, 143. 103679. https://doi.org/10.1016/j.compedu.2019.103679

Pérez, A., \& Rodríguez, M. J. (2016). Evaluación de las competencias digitales autopercibidas del profesorado de educación primaria en Castilla y León. Revista de Investigación Educativa, 34(2). 399-415. http://dx.doi.org/10.6018/rie.34.2.215121

Portillo, J., Garay, U., Tejada, E. \& Bilbao, N. (2020). Self-Perception of the Digital Competence of Educators during the COVID-19 Pandemic: A Cross-Analysis of Different Educational Stages. Sustainability, 12(23). 10128. https://doi.org/10.3390/su122310128

Pozos, K. V., \& Tejada, J. (2018). Competencias digitales en docentes de educación superior: niveles de dominio y necesidades formativas. Revista Digital de Investigación en Docencia Universitaria, 12(2). 59-87. http://dx.doi.org/10.19083/ridu.2018.712

Quezada, M. del P., Castro, M. del P., Oliva, J. M., Gallo, C. I. \& Quezada, G. A. (2020). Alfabetización digital como sustento del teletrabajo para docentes universitarios: hacia una sociedad inclusiva. Revista Conrado, 16(77). 332-337. Recuperado de: https://conrado.ucf.edu.cu/index.php/conrado/article/view/1604/1587

Ramirez, E. H., Espinoza, M. R., Esquivel, S. M. \& Naranjo, M. E. (2020). Inteligencia emocional, competencias y desempeño del docente universitario: Aplicando la técnica mínimos cuadrados parciales SEM-PLS. Revista Electrónica Interuniversitaria de Formación del Profesorado, 23(3). 99-114. https://doi.org/10.6018/reifop.428261

Reis, C., Pessoa, T. \& Gallego-Arrufat, M. J. (2019). Alfabetización y competencia digital en Educación Superior: Una revisión sistemática. REDU. Revista de Docencia Universitaria, 17(1). 45-58. https://doi.org/10.4995/redu.2019.11274 
Rodríguez, J., Ortiz, A. M., Cordón, E. \& Agreda, M. (2021). The Influence of Digital Tools and Social Networks on the Digital Competence of University Students during COVID-19 Pandemic. International Journal of Environmental Research and Public Health, 18(6). 2835. https://doi.org/10.3390/ijerph18062835

Rodríguez-García, A.M., Raso, F. \& Ruiz-Palmero, J. (2019). Competencia digital, educación superior y formación del profesorado: Un estudio de meta-análisis en la web of science. Pixel Bit. Rev. Medios Educ, 54. 65-81. https://doi.org/10.12795/pixelbit.2019.i54.04

Sánchez-Cruzado, C., Santiago, R. \& Sánchez, M. (2021). Teacher Digital Literacy: The Indisputable Challenge after COVID-19. Sustainability, 13(4). 1858. https://doi.org/10.3390/su13041858

Santana, J. S. \& Perez, A. (2020). Codiseño educativo haciendo uso de las TIC en educación superior una revisión sistemática de literatura. Edutec, Revista Electrónica De Tecnología Educativa, 74. 25-50. https://doi.org/10.21556/edutec.2020.74.1799

Spante, M., Hashemi, S. S., Lundin, M. L. \& Algers, A. (2018). Digital competence and digital literacy in higher education research: Systematic review of concept use. Cogent Education, 5(1). 1519143. https://doi.org/10.1080/2331186X.2018.1519143

Supo, J.A. \& Zacarias, H. R. (2020). Metodología de la investigación Científica: Para las Ciencias de la Salud y las Ciencias Sociales. Lima: Sociedad Hispana de Investigadores Cientificos, Sincie.

Terreni, L., Varas, G., \& Vilanova, J. (2019). Desarrollo de competencias digitales en propuestas pedagógicas en ambientes mediados: Un caso en educación superior bajo modelo de aula extendida. Informes Científicos Técnicos-UNPA, 11(3). 61-87. https://doi.org/10.22305/ict-unpa.v11.n3.797

UNESCO. (2008). Estándares de competencia en TIC para docentes. Londres: Organización de las Naciones Unidas para la Educación, la Ciencia y la Cultura, 1-28. Recuperado de: http:// www.eduteka.org/pdfdir/UNESCOEstandaresDocentes.pdf

Van Laar, E., Van Deursen, A. J., Van Dijk, J. A. \& De Haan, J. (2017). The relation between 21stcentury skills and digital skills: A systematic literature review. Computers in human behavior, 72. 577-588. https://doi.org/10.1016/j.chb.2017.03.010

Vargas-Murillo, G. (2019). Competencias digitales y su integración con herramientas tecnológicas en educación superior. Cuadernos Hospital de clínicas, 60(1). 88-94. Recuperado de: http://www.scielo.org.bo/scielo.php?pid=\$1652$67762019000100013 \&$ script $=$ sci arttext

Vega, U. J., Del Real, F. \& Figueroa, M. (2017). Reflexiones sobre la formación de competencias transversales y digitales en las instituciones de educación superior de México. Ensayos Pedagógicos, 12(1). 17-38. https://doi.org/10.15359/rep.12-1.1

Villarreal-Villa, S., García-Guliany, J., Hernández-Palma, H. \& Steffens-Sanabria, E. (2019). Competencias docentes y transformaciones en la educación en la era digital. Formación universitaria, 12(6). 3-14. http://dx.doi.org/10.4067/S071850062019000600003

Yslado, R. M., Ramirez, E. H., García, M. E. \& Arquero, J. L. (2021). Clima laboral y burnout en profesores universitarios. Revista Electrónica Interuniversitaria de Formación del Profesorado, 24(3). 101-114. https://doi.org/10.6018/reifop.476651 